\title{
Cerebral small vessel disease and systemic arteriopathy in intracranial arterial dolichoectasia patients
}

\author{
Fabio Fierini $^{1}$ | Anna Poggesi ${ }^{2}$ | Emilia Salvadori ${ }^{2}$ | Manlio Acquafresca ${ }^{3}$ | \\ Enrico Fainardi ${ }^{4}$ | Marco Moretti ${ }^{5}$ | Leonardo Pantoni ${ }^{6}$ (D)
}

${ }^{1}$ Psychiatry Unit, Department of Health Sciences, University of Florence, Florence, Italy

${ }^{2}$ NEUROFARBA Department, Neuroscience Section, University of Florence, Florence, Italy

${ }^{3}$ Radiology Unit 4, Department of Diagnostic Imaging, Careggi University Hospital,

Florence, Italy

${ }^{4}$ Neuroradiology Unit, Department of Experimental and Clinical Biomedical Sciences, University of Florence, Florence, Italy

${ }^{5}$ Neuroradiology Unit, Department of Diagnostic Imaging, Careggi University Hospital, Florence, Italy

${ }^{6}$ 'L. Sacco' Department of Biomedical and Clinical Sciences, University of Milan, Milan, Italy

\section{Correspondence}

Leonardo Pantoni, 'Luigi Sacco' Department of Biomedical and Clinical Sciences, University of Milan, Milan, Italy.

Email: leonardo.pantoni@unimi.it
Objectives: To investigate clinical and demographic characteristics of patients with intracranial arterial dolichoectasia (IADE) and describe the possible coexistence of cerebral small vessel disease (SVD) and systemic arteriopathy.

Material and methods: From January 2015 to March 2016, all the patients attending an outpatient service for chronic cerebrovascular diseases were screened for suspected IADE. Identified patients underwent a predefined protocol including: brain MR angiography for the diagnosis of IADE; brain MRI with visual rating of SVD features; whole-body CT angiography to assess signs of systemic arteriopathy; and neuropsychological examination.

Results: Among the 251 patients screened, IADE was diagnosed in seven (mean age \pm SD $68.8 \pm 7.2$ years, six males). Hypertension was the most frequent risk factor. All patients had basilar artery dolichoectasia, two also ectasia of a vessel of the anterior circulation. All patients had white matter hyperintensities that were moderate or severe in six, five had at least one lacune, and all had enlarged perivascular spaces. At least one microbleed was detected in six patients. A variable grade of global cortical atrophy was found in six patients. Systemic arterial ectasia was found in all but one patient. Neuropsychological examination showed a multidomain cognitive impairment in five patients.

Conclusions: Our study confirms the high prevalence of cerebral SVD in IADE. The involvement of the brain-supplying arteries is probably part of a systemic arteriopathy in IADE patients, thus suggesting the usefulness of assessing the whole arterial tree in clinical practice. Cognitive deterioration signs are frequent in these patients.

\section{KEYWORDS}

arteriopathy, cognitive function, computed tomography angiography, dolichoectasia,

magnetic resonance imaging, small vessel disease

\section{1 | INTRODUCTION}

Intracranial arterial dolichoectasia (IADE), also called dilatative arteriopathy of the brain vessels, is defined as an increase in the length and diameter of at least one intracranial artery. ${ }^{1}$ The prevalence of IADE is reported to be $0.1 \%-6.5 \%$ in the general population ${ }^{2}$ and about $12 \%$ among stroke patients, ${ }^{3}$ with the basilar artery involved in most cases. IADE may be asymptomatic or associated with stroke, subarachnoid hemorrhage, cranial nerve palsy, midbrain compression, or obstructive hydrocephalus.

To date, one of the most important observational studies focused on IADE is the Étude du Profil Génétique de I'Infarctus 
Cérébral (GENIC Study), in which the possible association with the main features of cerebral small vessel disease (SVD) and with enlarged systemic arteries was explored. ${ }^{1}$ Investigators found that among 510 consecutive patients with brain infarct, those having IADE more often showed multilacunar state, severe leukoaraiosis, and severe état criblé (enlarged perivascular spaces) on MRI. ${ }^{4}$ There was also a different distribution of stroke subtypes, being the lacunar stroke more frequent in patients with IADE than in patients without. ${ }^{3}$ A GENIC substudy showed that patients with IADE had larger descending thoracic aorta, supporting the hypothesis of a systemic nature of the process underlying IADE. ${ }^{5}$ Similar evidence comes from a recent autopsy series in which a significant association between IADE and coronary artery ectasia was found in stroke patients, suggesting a common pathogenesis. $^{6}$

In the context of an outpatient cerebrovascular disease clinic, we performed a prospective assessment of a small series of IADE patients with the aims to assess: (a) the demographic and clinical characteristics, including neuropsychological testing; (b) the coexistence of cerebral SVD; and (c) the presence of systemic vessel arteriopathy.

\section{2 | MATERIAL AND METHODS}

\section{1 | Clinical setting and study structure}

The study was conducted in accordance with the ethical principles of the Helsinki Declaration and involved outpatients referred to a clinic devoted to cerebrovascular diseases. ${ }^{7,8}$ Reasons for patient referral were, in particular: disturbances associated with the presence of cerebrovascular lesions or a history of stroke such as memory complaints, diagnosis of cognitive decline, language deficits, mood disorders, gait disturbances of the apraxic type; finding of vascular lesions on neuroimaging known to be possibly related to cognitive impairment; and screening for hereditary forms of cerebrovascular diseases.

From January 1, 2015, to March 1, 2016, out of the 251 patients attending the clinic, seven (2.8\%) were assigned a presumptive diagnosis of IADE using personal brain MRI based on the visual impression that the intracranial arteries were longer and wider than normal. Identified possible IADE patients underwent a protocol that included brain MR angiography to confirm the diagnosis of IADE according to the criteria proposed by Smoker et $\mathrm{al}^{9}$ and by Passero and Rossi ${ }^{10}$; family and clinical history assessment, with particular focus on stroke and vascular risk factor profile; extensive neuropsychological assessment; brain MRI, with visual rating of SVD features; and whole-body CT angiography to assess the presence of systemic arteriopathy. In accordance with usual clinical practice and based on the current diagnostic management, each patient underwent further instrumental and laboratory tests.

Informed consent was obtained from all the 251 patients.

\section{2 | Brain MR angiography and MRI protocol and assessment}

Brain MR angiography and MRI were performed using a Siemens 1.5 T Magnetom Aera machine, with a maximum gradient amplitude of $35 \mathrm{mT} / \mathrm{m}$ and a slew rate of $200 \mathrm{mT} \mathrm{m}^{-1} \mathrm{~ms}^{-1}$ The protocol included the following sequences: magnetization-prepared rapid gradient-echo (MPRAGE) 3D T1-weighed, axial fluid-attenuated inversion recovery (FLAIR) turbo spin-echo (TSE), axial T2-weighted TSE, axial T2-weighted gradient-echo (GE) fast low angle shot (FLASH), axial echo-planar diffusion-weighted imaging (EP-DWI), time-of-flight (TOF) MR angiography, and MR angiography with maximum intensity projection (MIP) multiplanar reconstructions. Two neurologists experienced in brain imaging assessment (FF and AP) reviewed all the available scans and assessed the following parameters: (a) presence of one or more dolichoectatic arteries, assessed by means of objective criteria proposed by Smoker et al $^{9}$ for basilar dolichoectasia (elongation indicated by the invasion of the dorsum sellae margin or by a bifurcation height located over the suprasellar cistern plane; ectasia indicated by a diameter cutoff $>4.5 \mathrm{~mm}$ as measured on TOF MR angiography sequences) and by Passero and Rossi ${ }^{10}$ for vessels other than basilar artery (ectasia indicated by diameter cutoffs for the internal carotid artery $\geq 7 \mathrm{~mm}$, middle cerebral artery $\geq 4 \mathrm{~mm}$, and vertebral artery $\geq 4 \mathrm{~mm}$, as measured on TOF sequences); (b) presence and severity of leukoencephalopathy (white matter hyperintensities on FLAIR or T2-weighted images) assessed by the modified Fazekas visual scale,$^{11}$ classifying all patients into three groups according to the grade of deep and subcortical white matter hyperintensity severity (grade 1 , mild white matter hyperintensities: single lesions $<10 \mathrm{~mm}$, or areas of "grouped" lesions $<20 \mathrm{~mm}$ in any diameter; grade 2 , moderate white matter hyperintensities: single hyperintense lesions between 10 and 20 mm, or areas of "grouped" lesions $\geq 20 \mathrm{~mm}$ in any diameter, no more than "connecting bridges" between individual lesions; and grade 3 , severe white matter hyperintensities: single lesions or confluent areas of hyperintensity $\geq 20 \mathrm{~mm}$ in any diameter); (c) presence and number of lacunes of presumed vascular origin, defined as focal hyperintensities on T2-weighted TSE images, $3 \mathrm{~mm}$ in size or larger, and with a corresponding hypointensity on T1weighted images, ${ }^{12}$ dividing patients into three groups according to the lacunes number (0, 1-3, or >3 lacunes); (d) number and location of enlarged perivascular spaces, defined as small ( $<3 \mathrm{~mm}$ wide), sharply delineated structures of cerebrospinal fluid intensity on T1-weighted images that follow the orientation of the perforating vessels and run perpendicular to the brain surface, dividing patients into five groups by means of the 5 -point scale proposed by Doubal et $\mathrm{al}^{13}(0=0$, $1=<10,2=11-20,3=21-40$, and $4=>40$, separated count for basal ganglia and semioval centrum; the numbers refer to enlarged perivascular spaces on one side of the brain; the higher score was used if there was asymmetry between the sides and enlarged perivascular spaces were counted in the slice with the highest number; basal ganglia and centrum semiovale enlarged perivascular spaces scores were summed to form a total score, 0 to 8 ); (e) number and location of microbleeds, defined as small, rounded or circular, well-defined 
hypointense lesions with clear margins ranging from 2 to $10 \mathrm{~mm}$ in size on T2-weighted GE images, using the Microbleed Anatomical Rating Scale ${ }^{14}$; and ( $f$ ) presence of global cortical atrophy, defined as a brain volume reduction that is not related to a specific macroscopic focal injury such as trauma or infarct, and classified into four groups (absent, mild, moderate, severe) by means of the Pasquier scale. ${ }^{15}$

The assessment of neuroimaging SVD features was also performed in patients not suspected of IADE using available images. The presence of SVD was defined as a modified Fazekas scale score $\geq 2$ or $=1$ plus one or more lacune.

\section{3 | Whole-body CT angiography protocol and assessment}

Systemic arteries CT angiography was performed using the Siemens SOMATOM Definition Flash machine, Dual Source technology, CARE platform, based on FLASH acquisition technique. The protocol included the following parameters: tomogram length 1970 mm, collimation $128 \times 0.6 \mathrm{~mm}$, spatial resolution $0.3 \mathrm{~mm}$, scan time $<0.2$ second, rotation time 0.28 second, tube settings $120 \mathrm{kV}, 426 \mathrm{mAs}$, and efficacious dose $3.7 \mathrm{mSv}$. Axial $0.6 \mathrm{~mm}$ thick images were obtained and processed using the syngo.via software. The lomeron $400 \mathrm{mg} / \mathrm{mL}$ Bracco contrast medium was administered by means of an automatic injector with a dosage of $100 \mathrm{~mL}$ (infusion speed $5.0 \mathrm{~mL} / \mathrm{s}$ ) prior preMonitor scansion with a $150 \mathrm{UH}$ threshold on the ascending aorta. The presence of atheromatous intimal thickening, ectasia, stenosis, and endoluminal occlusion was extensively investigated. Normative reference diameters were the following, for each major arterial district: aortic bulb 38-40 mm and ascending aorta $40 \mathrm{~mm}$ (adjusted for age and gender), aortic arch $35 \mathrm{~mm}$, descending aorta $28-30 \mathrm{~mm}$, suprarenal abdominal aorta $25 \mathrm{~mm}$, subrenal abdominal aorta $20 \mathrm{~mm}$, common iliac artery $15 \mathrm{~mm}$, internal iliac artery $8 \mathrm{~mm}$, external iliac artery $8-10 \mathrm{~mm}$, femoral artery 6-8 $\mathrm{mm}$, and popliteal artery $4-6 \mathrm{~mm}$.

\subsection{Cognitive function assessment}

Cognitive global efficiency was assessed by means of the MiniMental State Examination. Verbal memory was assessed by means of the Rey Auditory-Verbal Learning Test, a tool that includes an immediate and delayed recall after 15 minutes. In both tasks, the score was the total number of recalled words. Focalized and maintained attention was assessed by means of the Symbol Digit Modalities Test and a Visual Search test. The Symbol Digit Modalities Test is a symbol substitution task and assesses complex scanning and visual tracking. The Visual Search test requires visual selectivity at fast speed and assesses the capacity for sustained attention and accuracy of visual scanning. Divided and selective attention was assessed by means of the Trail Making Test and Color Word Stroop Test. The Trail Making Test is a test of complex visual scanning and divided attention and has an important motor speed component. The Color Word Stroop Test is a measure of concentration effectiveness and deals with response inhibition and selective attention. Finally, language was assessed by means of phonemic (P-F-L) and semantic (animals-fruits-cars) verbal fluency tests. For both tests, the final score was the total number of words produced.

\section{5 | Genetic assessment}

Based on clinical and neuroimaging features, three patients fit criteria for suspected Cerebral Autosomal Dominant Arteriopathy with Subcortical Infarcts and Leukoencephalopathy (CADASIL) ${ }^{16}$ and thus underwent genetic examination of the NOTCH3 gene (19p13). Because Fabry disease is an inherited disorder that has been reported in association with IADE, all the male patients were subjected to venous blood sampling for the assessment of blood level of alphagalactosidase activity, while in the female patient, a sequencing of the GLA gene (Xq22) was performed.

\section{3 | RESULTS}

A diagnosis of IADE was confirmed by consensus between two neurologists in all seven suspected patients. All patients had dolichoectasia of the basilar artery (mean diameter at midpons \pm SD $6.9 \pm 3.6 \mathrm{~mm}$ ), one also with carotid siphon ectasia, and one with carotid siphon and middle cerebral artery ectasia (Figure $1 \mathrm{~A}$ and 2 ).

The demographical characteristics and the vascular risk factor profile of the IADE and non-IADE patients are reported in Table 1. Among IADE patients, a personal history of stroke was reported in five cases. In particular, one suffered from a stroke due to a paramedian pontine infarct possibly caused by the occlusion of a penetrating artery by a thrombus located in the dolichoectatic basilar artery; one had a stroke due to multiple ischemic lesions in the bulbopontine junction and right frontotemporal white matter; two had a lacunar stroke related to small infarcts in the centrum semiovale; and one had a right temporal lobe hemorrhage of undetermined cause.

All IADE patients had white matter hyperintensities and enlarged perivascular spaces, five had at least one lacune, and six had at least one microbleed; a variable grade of global cortical atrophy was detected in six patients (Figure $1 \mathrm{~B}-\mathrm{H}$ and Table 2). Among non-IADE patients, the assessment of neuroimaging SVD features was performed in 241/244 cases (images were not available for three patients) and showed a SVD prevalence of $76.3 \%$.

Considering systemic circulation, six IADE patients had ectasia of at least one artery, while one did not have any other enlarged artery (Figures 2 and 3); all patients had atherosclerotic intimal thickening involving at least one arterial district, while two had stenosis or obstruction of at least one extracranial artery.

The neuropsychological examination revealed normal cognitive performances in all the investigated domains for two IADE patients and a variable grade of multidomain cognitive impairment for five (Table 3). Among the investigated domains, psychomotor speed, executive functions, and attention were the most frequently involved, with a partial preservation of long-term and especially short-term memory. No significant language dysfunctions emerged. 

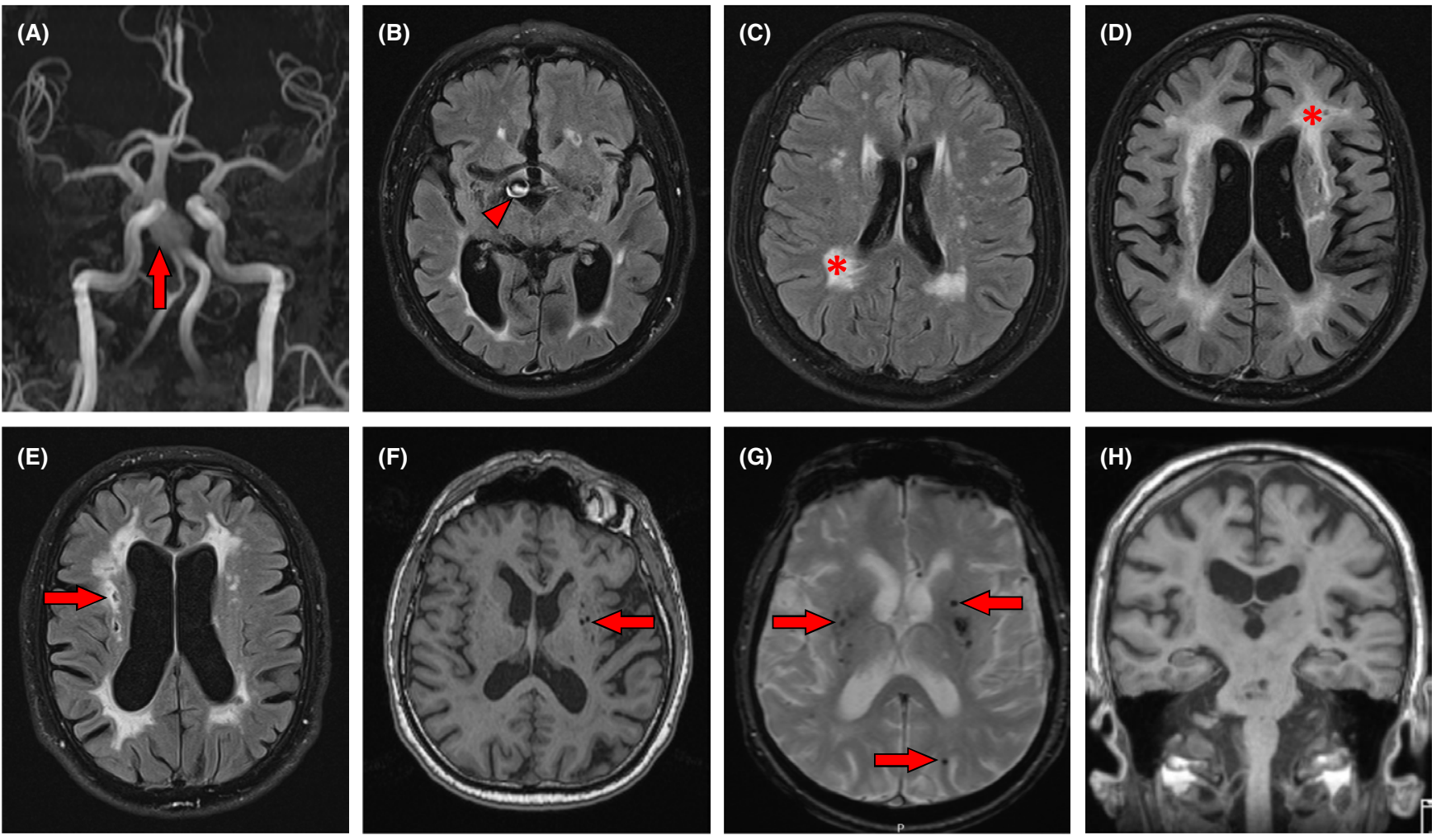

FIG URE 1 Brain MR angiography and MRI findings in the IADE sample. (A) Gadolinium MR angiography showing basilar artery dolichoectasia (arrow) in Patient 2. (B, C, D, and E) Axial fluid-attenuated inversion recovery (FLAIR) MRI showing enlarged basilar artery (B; arrowhead) in Patient 4, deep and periventricular white matter hyperintensities ( $C$ and $D$; asterisk) in Patients 7 and 1 , and lacunes ( $E$; arrow) in Patient 4. (F) Axial T1-weighted MRI showing enlarged perivascular spaces in the basal ganglia (arrow) in Patient 2. (G) Axial T2-weighted gradient-echo (GE) MRI showing deep and lobar cerebral microbleeds (arrows) in Patient 3. (H) Coronal T1-weighted magnetization-prepared rapid gradient-echo (MPRAGE) MRI showing global cortical atrophy in Patient 6
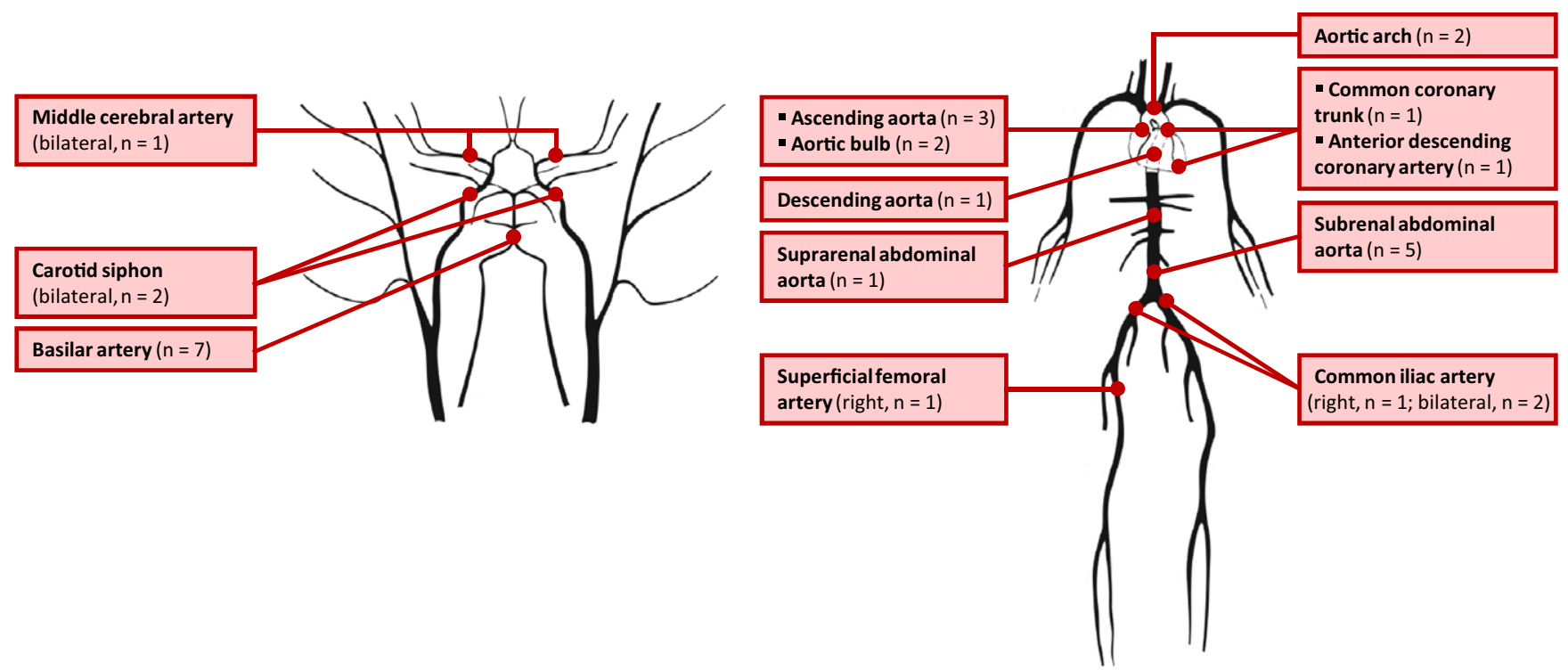

FIGURE 2 Arterial ectasia distribution for anatomical district in the IADE sample. Left panel: detailed representation of the brain arterial tree, highlighting vascular ectasia and dolichoectasia side and location. Right panel: detailed representation of the systemic arterial tree, highlighting vascular ectasia side and location. Based on the model of Stergiopulos et $\mathrm{al}^{33}, \mathrm{n}$ indicates number of patients

Regarding the genetic assessment, the NOTCH3 gene sequencing did not lead to the identification of known pathogenic mutations responsible for CADASIL in the IADE sample. The GLA gene analysis did not highlight pathogenic mutations in the female patient, while the assay of the alpha-galactosidase activity was found within normal limits in the six males. 


\begin{tabular}{|lll|}
\hline Characteristics & IADE patients & Non-IADE patients \\
$\mathrm{n}=7$ & $\mathrm{n}=244$
\end{tabular}

TABLE 1 Demographic characteristics and vascular risk factor profile in IADE and non-IADE patients

IADE, intracranial arterial dolichoectasia; SD, standard deviation; n, number of patients.

\begin{tabular}{|c|c|c|c|c|c|c|c|}
\hline \multirow[b]{2}{*}{ Variables } & \multicolumn{7}{|c|}{ Patient } \\
\hline & 1 & 2 & 3 & 4 & 5 & 6 & 7 \\
\hline \multicolumn{8}{|l|}{ IADE } \\
\hline Basilar artery, diameter (mm) & 5.9 & 15.5 & 6.8 & 5.8 & 4.9 & 4.7 & 4.8 \\
\hline Anterior circulation involvement & $\mathrm{N}$ & Y & $\mathrm{N}$ & Y & $\mathrm{N}$ & $\mathrm{N}$ & $\mathrm{N}$ \\
\hline $\begin{array}{l}\text { White matter hyperintensities } \\
\text { (grade) }^{\mathrm{a}}\end{array}$ & 3 & 1 & 2 & 3 & 2 & 2 & 3 \\
\hline Lacunes (number) & $>3$ & $>3$ & $1-3$ & $>3$ & $>3$ & 0 & 0 \\
\hline \multicolumn{8}{|l|}{ Enlarged perivascular spaces (grade) ${ }^{\mathrm{b}}$} \\
\hline Basal ganglia & 4 & 2 & 2 & 1 & 2 & 3 & 1 \\
\hline Centrum semiovale & 4 & 0 & 0 & 0 & 0 & 0 & 0 \\
\hline Total & $8 / 8$ & $2 / 8$ & $2 / 8$ & $1 / 8$ & $2 / 8$ & $3 / 8$ & $1 / 8$ \\
\hline Midbrain & 1 & 0 & 1 & 1 & 0 & 0 & 0 \\
\hline Hippocampus & 1 & 0 & 1 & 1 & 1 & 1 & 1 \\
\hline \multicolumn{8}{|l|}{ Cerebral microbleeds (number) ${ }^{c}$} \\
\hline \multicolumn{8}{|l|}{ Definite } \\
\hline Lobar & 1 & 0 & 11 & 1 & 2 & 1 & 0 \\
\hline Deep & 1 & 0 & 16 & 2 & 0 & 1 & 5 \\
\hline Subtentorial & 0 & 0 & 10 & 0 & 0 & 0 & 0 \\
\hline \multicolumn{8}{|l|}{ Possible } \\
\hline Lobar & 1 & 0 & 8 & 0 & 6 & 0 & 0 \\
\hline Deep & 1 & 0 & 5 & 2 & 0 & 0 & 2 \\
\hline Subtentorial & 0 & 0 & 4 & 0 & 0 & 0 & 0 \\
\hline Global cortical atrophy (grade) ${ }^{\mathrm{d}}$ & 3 & 1 & 2 & 2 & 2 & 2 & 0 \\
\hline
\end{tabular}

TAB LE 2 Brain MR angiography and MRI findings in the IADE sample

IADE, intracranial arterial dolichoectasia; $\mathrm{Y}$, yes; $\mathrm{N}$, no.

${ }^{a}$ Assessed with the modified Fazekas scale.

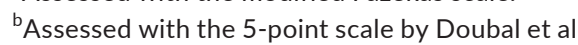

${ }^{\mathrm{c}}$ Assessed with the Microbleed Anatomical Rating Scale.

${ }^{\mathrm{d}}$ Assessed with the Pasquier scale.

\section{4 | DISCUSSION}

In our series, we made a diagnosis of IADE in $2.8 \%$ of the total number of screened patients in the reference period. This finding is quite in line with the estimate of IADE prevalence in the general population.
Patients with IADE had a mean age of about 69 years, were more frequently males, and all had hypertension. A high frequency of older age, male sex, and a history of hypertension was noted in IADE patients in other series without control groups. ${ }^{17-20}$ In this regard, in the GENIC Study, a significant positive association emerged between IADE and 

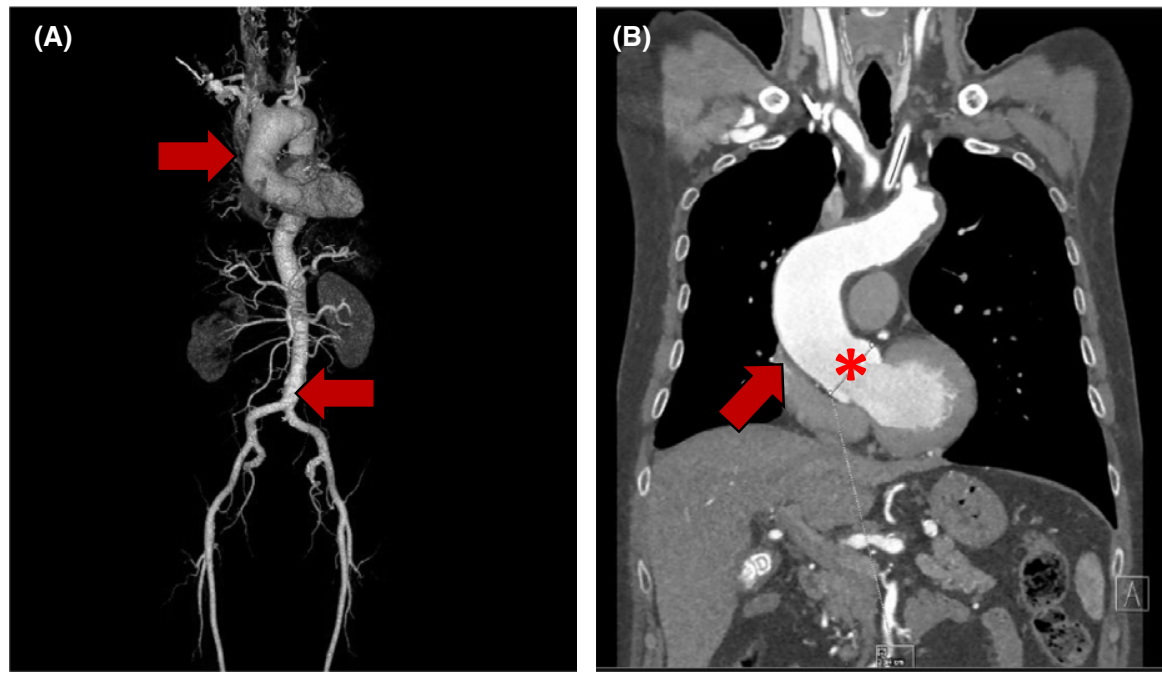

FIGURE 3 Whole-body CT angiography findings in the IADE sample. (A) 3D reconstruction $C T$ angiography showing ascending aorta, aortic arch, and abdominal aorta ectasia (arrows) in Patient 2. (B) CT angiography (frontal view): detail of aortic bulb (asterisk), ascending aorta, and aortic arch ectasia (arrow) in Patient 3

TAB LE 3 Neuropsychological assessment in the IADE sample

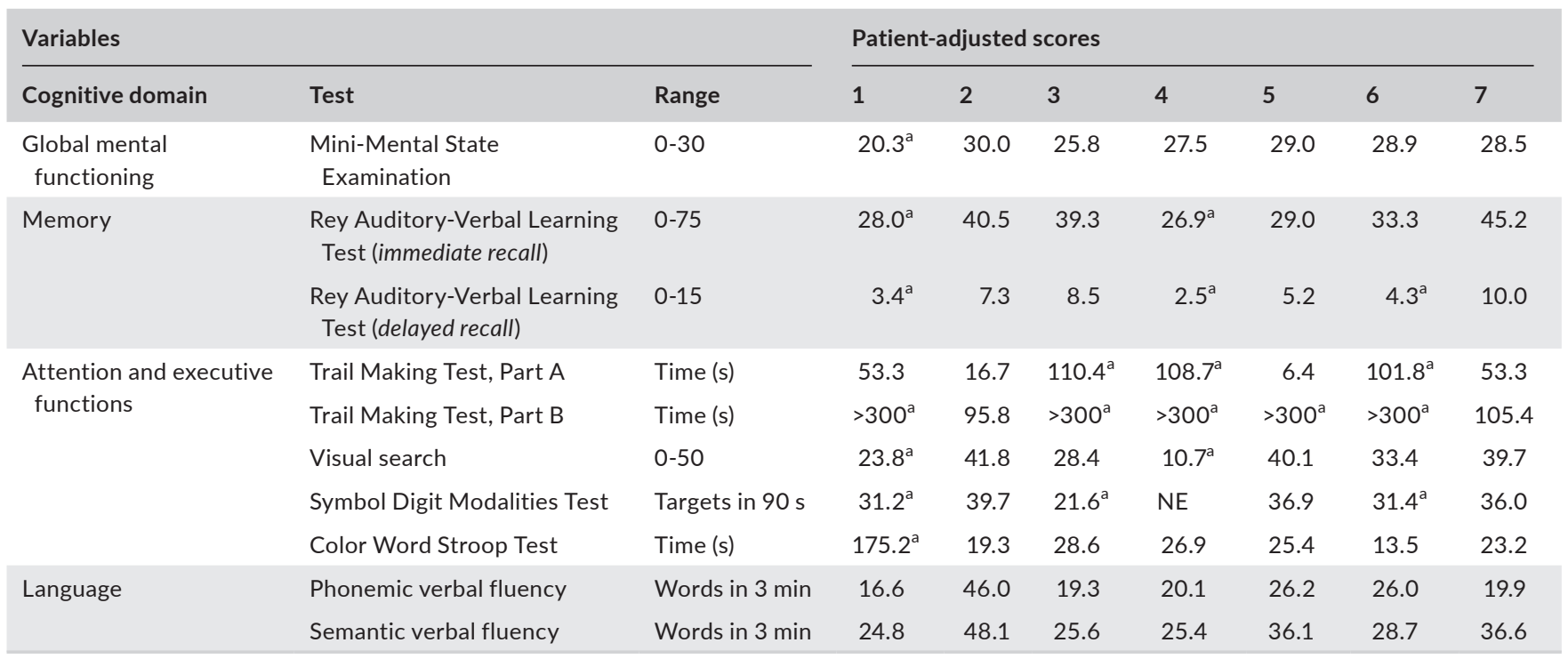

Raw scores were adjusted according to normative data on healthy Italian adult samples. $\mathrm{NE}$, not evaluable.

${ }^{a}$ Abnormal performance, that is, lower than the 5 th percentile of the normal population.

older age, male sex, and hypertension, ${ }^{3}$ suggesting a role of these factors in the pathogenesis of the dolichoectatic process. ${ }^{1}$ Other traditional factors such as increased body mass index, cigarette smoking, dyslipidemia, and diabetes mellitus show conflicting results. ${ }^{3,21,22}$

The dolichoectatic process affected the posterior circulation alone in five patients and both the posterior and anterior circulation in two patients. In the GENIC Study, IADE involved the posterior circulation (alone or in combination with the anterior circulation) in $87 \%$ of cases, while the basilar artery resulted dolichoectatic in $78 \%$ of patients. ${ }^{3}$ In another case-control study conducted on 387 patients with brain infarct in which CT scan was the main imaging method, the dolichoectatic process involved the posterior circulation in $83 \%$ of IADE patients. ${ }^{23}$

We reported a relevant presence of cerebral SVD features in our sample. In line with the GENIC Study results, ${ }^{4}$ our patients with IADE more often had SVD features than patients without. In a recent MRI-based study, basilar artery dolichoectasia was associated with both white matter hyperintensities and cerebral microbleeds. ${ }^{24}$ Similarly, in a series of 187 patients hospitalized because of ischemic stroke or transient ischemic attack, microbleeds were more prevalent in stroke patients with IADE than in those without, especially in the posterior circulation territory. ${ }^{25}$ Regarding the hypothesized association between IADE and cerebral SVD, some considerations can be put forward. First, our data suggest that IADE and SVD may share some undetermined risk factors (environmental and/or genetic). Supposed interactions between common vascular risk factors and a predisposing genotype might impair the matrix metalloproteinase balance in the tunica media contributing to IADE pathophysiology, similarly to what is observed in SVD. ${ }^{26,27}$ Second, IADE and SVD could be 
different phenotypic expression of the same pathophysiological mechanisms, respectively involving macro- and microvessels. The hypothesis that the dolichoectatic process may also affect the small vessels of the brain was first suggested in a post-mortem MRI-based study in which all areas with myelin staining pallor, corresponding to MRI white matter hyperintensities, showed a spectrum of alterations ranging from arteriosclerosis to ectasia of small perforating arteries and veins. ${ }^{28}$

In our series, the majority of patients exhibited ectasia of at least one extracranial artery. The thoracoabdominal was the most frequently involved district. To the best of our knowledge, this is the first prospective study thoroughly investigating the correlation between IADE and systemic arteriopathy by means of whole-body CT angiography. However, the reported findings are not of univocal interpretation from a pathogenic point of view and need to be confirmed in larger patient series. One autopsy study based on 34 IADE patients found an abdominal aortic aneurysm in 12 cases but, as in our study, there was no control group. ${ }^{29}$ Other uncontrolled studies on IADE patients that used autopsy and angiography material have occasionally found some arterial abnormalities, such as saccular intracranial aneurysms, dolichoectasia of the cervical arteries, and iliac arteries aneurysms. ${ }^{17,18,29}$ In a substudy of the GENIC, there was a clear association between IADE and diameter of the descending thoracic aorta measured by transesophageal echocardiography. ${ }^{5}$ Furthermore, the GENIC investigators found that in a large autopsy series based on stroke patients, there was a significant association between IADE and coronary artery ectasia. ${ }^{6}$ Fragmentation of the internal elastic lamina caused by increased plasma concentrations of some metalloproteinases and/or decreased concentrations of their inhibitors has been reported in patients with either abdominal aortic aneurysms or IADE. ${ }^{26}$ Likewise, a proteolytic imbalance induced by specific metalloproteinase polymorphisms has also been related to the development of thoracic aorta dissection and coronary artery ectasia. ${ }^{30,31}$

In our patients, the presence of intimal thickening and plaques with or without stenoses likely reflects the coexistence of IADE and systemic atherosclerosis. In the past, due to the fact that IADE and atherosclerosis share similar risk factors, these disorders were postulated to have a common pathogenesis. Although the preferential development of atherosclerotic plaques in response to hemodynamic changes induced by dolichoectasia reinforced the assumption of IADE as a severe form of atherosclerosis, further investigations definitively pointed out different pathophysiological pathways. ${ }^{2}$ Whereas IADE involves disruption of the elastic tissue of the tunica media and of the internal elastic lamina, atherosclerosis involves oxidized lipid infiltration and inflammation of the vascular wall intima. ${ }^{1}$ In the GENIC Study, the lack of any association between IADE and several ultrasonographic markers of atherosclerosis such as common carotid arteries intima-media thickness and carotid plaques corroborated on clinical grounds the hypothesis that IADE is not an ectatic variant of atherothrombotic disease. ${ }^{3}$

We recorded a high prevalence of neuropsychological deficits in our small series. From our data, it is not possible to establish whether this finding is more related with the disease of the large arteries or with the almost invariably associated cerebral SVD. While various biomarkers of SVD have been put in relationship with cognitive impairment, some recent data seem to support also the association between larger brain arterial diameter and lower performances on neuropsychological tests regardless the occurrence of major vascular events. ${ }^{32}$

This study has limitations. First, the nature of the study is uncontrolled, and therefore, it is not possible to draw definitive conclusions about IADE and its association with cerebral SVD and systemic arteriopathy. Second, the sample size is limited in number and lacks of heterogeneity, being composed by "somehow selected" patients with a high frequency of sporadic or familiar forms of cerebral microangiopathy.

With the above-mentioned limitations in mind, our data seem to support however that in patients with IADE, the study of systemic vessels and cognitive profile is warranted. For the first one, the use of recent $\mathrm{CT}$ protocols and machines can allow the examination to be performed in only one session and reduce the amount of infused contrast medium. Concerning the second, our study reinforces the idea that neuropsychological testing should be performed systematically in patients with cerebrovascular diseases also in the absence of overt stroke. Future studies performed with larger sample will allow to assess whether the neuropsychological deficits found in IADE are more related to the larger arterial diameter or the coexistence of cerebral SVD.

\section{ACKNOWLEDGEMENTS}

The authors would like to thank anonymous reviewers whose comments contributed to improve the manuscript.

\section{CONFLICT OF INTEREST}

The authors declare that they have no conflict of interest.

\section{ORCID}

Leonardo Pantoni iD http://orcid.org/0000-0001-7357-8530

\section{REFERENCES}

1. Pico F, Labreuche J, Amarenco P. Pathophysiology, presentation, prognosis, and management of intracranial arterial dolichoectasia. Lancet Neurol. 2015;14:833-845.

2. Del Brutto VJ, Ortiz JG, Biller J. Intracranial arterial dolichoectasia. Front Neurol. 2017;8:344.

3. Pico F, Labreuche J, Touboul PJ, Amarenco P. Intracranial arterial dolichoectasia and its relation with atherosclerosis and stroke subtype. Neurology. 2003;61:1736-1742.

4. Pico F, Labreuche J, Touboul PJ, Leys D, Amarenco P. Intracranial arterial dolichoectasia and small-vessel disease in stroke patients. Ann Neurol. 2005;57:472-479.

5. Pico F, Labreuche J, Cohen A, Touboul PJ, Amarenco P. Intracranial arterial dolichoectasia is associated with enlarged descending thoracic aorta. Neurology. 2004;63:2016-2021. 
6. Pico F, Labreuche J, Hauw JJ, Seilhean D, Duyckaerts C, Amarenco P. Coronary and basilar artery ectasia are associated: results from an autopsy case-control study. Stroke. 2016;47:224-227.

7. Ciolli L, Poggesi A, Salvadori E, et al. The VAS-COG clinic: an out-patient service for patients with cognitive and behavioral consequences of cerebrovascular diseases. Neurol Sci. 2012;33:1277-1283.

8. Poggesi A, Salvadori E, Valenti R, et al. The Florence VAS-COG clinic: a model for the care of patients with cognitive and behavioral disturbances consequent to cerebrovascular diseases. J Alzheimers Dis. 2014;42(Suppl 4):S453-S461.

9. Smoker WR, Price MJ, Keyes WD, Corbett JJ, Gentry LR. High-resolution computed tomography of the basilar artery: 1. Normal size and position. AJNR Am J Neuroradiol. 1986;7:55-60.

10. Passero SG, Rossi S. Natural history of vertebrobasilar dolichoectasia. Neurology. 2008;70:66-72.

11. Fazekas F, Chawluk JB, Alavi A, Hurtig HI, Zimmerman RA. MR signal abnormalities at 1.5 T in Alzheimer's dementia and normal aging. AJR Am J Roentgenol. 1987;149:351-356.

12. Gouw AA, van der Flier WM, Pantoni L, et al. LADIS study group. On the etiology of incident brain lacunes: longitudinal observations from the LADIS study. Stroke. 2008;39:3083-3085.

13. Doubal FN, MacLullich AM, Ferguson KJ, Dennis MS, Wardlaw JM. Enlarged perivascular spaces on MRI are a feature of cerebral small vessel disease. Stroke. 2010;41:450-454.

14. Gregoire SM, Chaudhary UJ, Brown MM, et al. The Microbleed Anatomical Rating Scale (MARS): reliability of a tool to map brain microbleeds. Neurology. 2009;73:1759-1766.

15. Pasquier F, Leys D, Weerts JG, Mounier-Vehier F, Barkhof F, Scheltens P. Inter- and intraobserver reproducibility of cerebral atrophy assessment on MRI scans with hemispheric infarcts. Eur Neurol. 1996;36:268-272.

16. Pescini F, Nannucci S, Bertaccini B, et al. The cerebral autosomaldominant arteriopathy with subcortical infarcts and leukoencephalopathy (CADASIL) scale: a screening tool to select patients for NOTCH3 gene analysis. Stroke. 2012;43:2871-2876.

17. Yu YL, Moseley IF, Pullicino P, McDonald WI. The clinical picture of ectasia of the intracerebral arteries. J Neurol Neurosurg Psychiatry. 1982;45:29-36.

18. Nishizaki T, Tamaki N, Takeda N, Shirakuni T, Kondoh T, Matsumoto S. Dolichoectatic basilar artery: a review of 23 cases. Stroke. 1986;17:1277-1281.

19. Milandre L, Bonnefoi B, Pestre P, Pellissier JF, Grisoli F, Khalil R. Vertebrobasilar arterial dolichoectasia. Complications and prognosis. Rev Neurol (Paris). 1991;147:714-722.

20. Nijensohn DE, Saez RJ, Reagan TJ. Clinical significance of basilar artery aneurysms. Neurology. 1974;24:301-305.

21. Gutierrez J, Bagci A, Gardener H, et al. Dolichoectasia diagnostic methods in a multi-ethnic, stroke-free cohort: results from the northern Manhattan study. J Neuroimaging. 2014;24:226-231.
22. Nakajima M, Pearce LA, Ohara $\mathrm{N}$, et al. SPS3 Investigators. Vertebrobasilar ectasia in patients with lacunar stroke: the secondary prevention of small subcortical strokes trial. J Stroke Cerebrovasc Dis. 2015;24:1052-1058.

23. Ince B, Petty GW, Brown RD Jr, Chu CP, Sicks JD, Whisnant JP. Dolichoectasia of the intracranial arteries in patients with first ischemic stroke: a population-based study. Neurology. 1998;50:1694-1698.

24. Del Brutto OH, Mera RM, Del Brutto VJ, Costa AF, Zambrano M, Brorson J. Basilar artery dolichoectasia: prevalence and correlates with markers of cerebral small vessel disease in community-dwelling older adults. J Stroke Cerebrovasc Dis. 2017;26:2909-2914.

25. Park JM, Koo JS, Kim BK, et al. Vertebrobasilar dolichoectasia as a risk factor for cerebral microbleeds. Eur J Neurol. 2013;20:824-830.

26. Pico F, Jacob MP, Labreuche J, et al. Matrix metalloproteinase-3 and intracranial arterial dolichoectasia. Ann Neurol. 2010;67:508-515.

27. Rosenberg GA, Sullivan N, Esiri MM. White matter damage is associated with matrix metalloproteinases in vascular dementia. Stroke. 2001;32:1162-1168.

28. Awad IA, Johnson PC, Spetzler RF, Hodak JA. Incidental subcortical lesions identified on magnetic resonance imaging in the elderly. II. Postmortem pathological correlations. Stroke. 1986;17:1090-1097.

29. Sacks JG, Lindenburg R. Dolicho-ectatic intracranial arteries: symptomatology and pathogenesis of arterial elongation and distention. Johns Hopkins Med J. 1969;125:95-106.

30. Liu O, Xie W, Qin Y, et al. MMP-2 gene polymorphisms are associated with type A aortic dissection and aortic diameters in patients. Medicine (Baltimore). 2016;95:5175.

31. Lamblin N, Bauters C, Hermant X, Lablanche JM, Helbecque N, Amouyel P. Polymorphisms in the promoter regions of MMP-2, MMP-3, MMP-9 and MMP-12 genes as determinants of aneurysmal coronary artery disease. J Am Coll Cardiol. 2002;40:43-48.

32. Gutierrez J, Kulick E, Park Moon Y, et al. Brain arterial diameters and cognitive performance: the Northern Manhattan study. J Int Neuropsychol Soc. 2018;24:335-346.

33. Stergiopulos N, Young DF, Rogge TR. Computer simulation of arterial flow with applications to arterial and aortic stenosis. J Biomech. 1992;25:1477-1488.

How to cite this article: Fierini F, Poggesi A, Salvadori E, et al. Cerebral small vessel disease and systemic arteriopathy in intracranial arterial dolichoectasia patients. Acta Neurol Scand. 2019;139:150-157. https://doi.org/10.1111/ane.13038 